\title{
Exact results in a slave boson saddle point approach for a strongly correlated electron model
}

\author{
Raymond Frésard ${ }^{1}$ and Thilo Kopp ${ }^{2}$ \\ ${ }^{1}$ Laboratoire CRISMAT UMR CNRS-ENSICAEN 6508, and IRMA, FR3095, Caen, France \\ ${ }^{2}$ Center for Electronic Correlations and Magnetism, \\ Institute of Physics, Universität Augsburg, D-86135 Augsburg, Germany
}

(Dated: November 29, 2018)

\begin{abstract}
We revisit the Kotliar-Ruckenstein (KR) slave boson saddle point evaluation for a two-site correlated electron model. As the model can be solved analytically, it is possible to compare the KR saddle point results to the exact many particle levels. The considered two site cluster mimics an infinite- $U$ single-impurity Anderson model with a nearest neighbor Coulomb interaction: one site is strongly correlated with an infinite local Coulomb repulsion which hybridizes with the second site, on which the local Coulomb repulsion vanishes. Making use of the flexibility of the representation we introduce appropriate weight factors in the KR saddle point scheme. Ground state and all excitation levels agree with the exact diagonalization results. Thermodynamics and correlation functions may be recovered in a suitably renormalized saddle point evaluation.

PACS numbers: 11.15.Tk, 71.27.+a, 11.15.Me

Keywords:
\end{abstract}

The unusual properties of the high temperature superconductors or the colossal magnetoresistance and orbital ordering in transition metal oxides $\underline{1}$ are calling for new techniques and concepts to describe these phenomena. A formalism, devised to deal specifically with strongly correlated electron systems, is provided by the slave boson approach pioneered by Barnes ${ }^{2}$ for the single impurity Anderson model (SIAM), and further extended to the Hubbard model by Kotliar and Ruckenstein $\underline{\underline{3}}^{\mathrm{T}}$ The approach implements a local decomposition of electronic excitations into charge and spin components which is achieved by the introduction of composite operators for all local (on-site) excitations. The composite operators separate into canonical bosonic and fermionic operators where, in the Barnes approach, the charge (spin) degrees of freedom are represented by bosonic (fermionic) operators. However the latter are enslaved in the sense that their respective number operators have to fulfill a local constraint. The original idea was to decouple spin and charge degrees of freedom. In the KotliarRuckenstein (KR) scheme a bosonic mode is attributed to each type of excitation which allows to study the correlated system in a saddle point approximation for all degrees of freedom.,$\frac{3.4}{5}$ This latter approach has been impressively successful when compared to numerical simulations: ground state energies ${ }^{6}$ and charge structure factors show excellent agreement ${ }^{\underline{T}}$

The slave boson approach has several intriguing properties. Firstly, the approach is exact in the large degeneracy limit. 4.8 Moreover, the paramagnetic meanfield solution reproduces the Gutzwiller approximation in the KR representation ${ }^{\frac{3}{2}}$ It obeys a variational principle in the limit of large spatial dimensions where the Gutzwiller approximation and the Gutzwiller wave function are identical. $\stackrel{9}{ }$ These formal properties signify that the approach captures characteristic features of strongly correlated electrons as the suppression of the quasi- particle weight and the Mott-Hubbard/Brinkman-Rice transition $\underline{10}$ to an insulating state at half filling with increasing on-site Coulomb interaction.

Secondly, as the fields are canonical within this approach, long range correlations are more amenable to analyses. Moreover, one can easily introduce a long range Coulomb interaction. In a radial gauge representation of the slave bosons, long range Coulomb interactions can even be cast into a bilinear form $\stackrel{11}{=}$ In other approaches, which satisfactorily implement local correlations, long range Coulomb interactions are typically difficult to handle.

The flexibility of the slave boson approach has contributed to its success in many fields from Kondo physics 12,13 and Kondo/Anderson lattices $14,15,16$ to interfaces of correlated electronic systems $\frac{17,18}{}$ It separates in a straightforward way the high and low energy scales. By now the respective mean-field evaluations are well documented (see, e.g., Refs. 6 19,20,21]), and a number of fluctuation calculations have been performed,,$\frac{7,23,24,25}{2}$ even though the choice of the proper framework has been intensely debated $\stackrel{4,11,23,26,27}{ }$ In the case of the single impurity Anderson model (SIAM) one rather resorts to diagrammatic approaches which include slave boson techniques (for a recent reference see Ref. [28]).

It was doubted that the low energy physics is implemented consistently in the mean field evaluations because the decomposition is a local scheme. In the present work we want to access all energy scales for a specific model and compare the saddle point evaluation of the KR slave boson approach to exact results. Comparing exact and saddle point calculations analytically represents a difficult task when handling a large system. Therefore, as a first step, we restrict our considerations to a two-site cluster which can be diagonalized exactly but still incorporates characteristics of a strongly correlated electron system: the considered two site cluster presents a truncated 
infinite- $U$ single-impurity Anderson model with a nearest neighbor Coulomb interaction. One site is strongly correlated with an infinite local Coulomb repulsion which hybridizes with the second site, on which the local Coulomb repulsion vanishes. From the investigation of this model another benchmark of the slave boson approach is provided here and, moreover, two essential questions may be addressed: how do thermodynamics and correlation functions compare between exact and saddle point evaluation? How is the intersite Coulomb interaction appropriately introduced in the KR slave boson approach?

Interacting two-site cluster. We introduce the SIAMtype Hamiltonian

$$
\begin{aligned}
\mathcal{H}= & \sum_{\sigma}\left(\epsilon_{\mathrm{c}} c_{\sigma}^{\dagger} c_{\sigma}+\epsilon_{\mathrm{d}} d_{\sigma}^{\dagger} d_{\sigma}+V\left(c_{\sigma}^{\dagger} d_{\sigma}+\text { h.c. }\right)\right) \\
& +U d_{\uparrow}^{\dagger} d_{\uparrow} d_{\downarrow}^{\dagger} d_{\downarrow}+I n_{\mathrm{d}} n_{\mathrm{c}}
\end{aligned}
$$

which is defined on two sites. The operators $c_{\sigma}^{\dagger}\left(c_{\sigma}\right)$ and $d_{\sigma}^{\dagger}\left(d_{\sigma}\right)$ represent the creation (annihilation) of "band" and "impurity" electrons, with spin projection $\sigma$. The energies $\epsilon_{\mathrm{c}}$ and $\epsilon_{\mathrm{d}}$ are the band and impurity energy levels, respectively, while the hybridization energy is $V$. Here $U$ is the on-site repulsion, which is the largest energy scale in the model and it will be set to infinity as in the standard infinite- $U$ SIAM. Finally, $\mathcal{H}_{I} \equiv I n_{\mathrm{d}} n_{\mathrm{c}}$ represents the non-local Coulomb interaction.

This extended two-site SIAM may be solved exactly, either by diagonalization of the Hamiltonian, Eq. (11), or, in the Lagrangian language, $\stackrel{29,30}{,}$ through the exact evaluation of the path integrals representing the desired quantities within a slave boson representation in the radial gauge ${ }^{11}$ The two-particle basis for the Hamiltonian matrix consists of two singlet states and three triplet states. Indeed one finds a threefold degenerate eigenvalue of $\mathcal{H}$

$$
E^{(t)}=\left(\epsilon_{\mathrm{c}}+\epsilon_{\mathrm{d}}+I\right)
$$

corresponding to the triplet states, and two nondegenerate eigenvalues controlled through $\Delta \equiv \epsilon_{\mathrm{c}}-\epsilon_{\mathrm{d}}$ :

$$
E_{ \pm}^{(s)}=\frac{1}{2}\left(3 \epsilon_{\mathrm{c}}+\epsilon_{\mathrm{d}}+I \pm \sqrt{(\Delta-I)^{2}+8 V^{2}}\right)
$$

corresponding to the singlet states.

$K R$ slave boson scheme. The goal is now to formulate an effective theory which correctly reproduces the expectation value of the charge density and the charge and spin density correlation functions. The requirement to work with canonical fermionic or bosonic operators (or rather 'fields' in the Lagrangian language) leads to the slave boson scheme with which we intend to evaluate the free energy in the KR SBMF (slave boson mean field or, equivalently, saddle point) approximation. The first step in the scheme is to enlarge the Fock space for the impurity site by introducing auxiliary fermions $f_{\sigma}^{(\dagger)}$ and slave bosons $e^{(\dagger)}$ and $p_{\sigma}^{(\dagger)}$, where $e^{(\dagger)}$, acts on an empty and $p_{\sigma}^{(\dagger)}$ on a singly occupied impurity site. A field for double occupancy of the impurity site is omitted as we restrict the evaluation to infinite $U$. The electron operator on the impurity is then represented as a composite operator

$$
d_{\sigma}^{\dagger}=f_{\sigma}^{\dagger} p_{\sigma}^{\dagger} e, \quad d_{\sigma}=e^{\dagger} p_{\sigma} f_{\sigma} .
$$

These fields are subject to three constraints:

$$
\begin{gathered}
e^{\dagger} e+\sum_{\sigma} p_{\sigma}^{\dagger} p_{\sigma}=1 \\
p_{\sigma}^{\dagger} p_{\sigma}=f_{\sigma}^{\dagger} f_{\sigma} \quad \sigma=\uparrow, \downarrow
\end{gathered}
$$

which are enforced by three Lagrange multipliers, denoted by $\alpha$ and $\lambda_{\sigma}$, respectively. If strictly enforced, only empty and singly occupied states in the physical space remain.

Now, in the second step of the setup, the KR technique renormalizes the coupling parameters of the Hamiltonian or Lagrangian. It thereby makes use of the freedom to choose different (operator) representations of the respective coupling terms, which are all equivalent in the physical subspace. This freedom originates from the decomposition of the electron field into auxiliary fields. Kotliar and Ruckenstein ${ }^{3}$ introduced $z$-factors into the hybridization term which then reads for the SIAM:

$$
\begin{aligned}
\mathcal{H}_{V} & =V \sum_{\sigma}\left(c_{\sigma}^{\dagger} d_{\sigma}+\text { h.c. }\right) \\
& =V \sum_{\sigma}\left(c_{\sigma}^{\dagger} z_{\sigma} f_{\sigma}+\text { h.c. }\right)
\end{aligned}
$$

A straightforward choice would be $z_{\sigma}=e^{\dagger} p_{\sigma}$ which directly translates the relation Eq. (4) within $\mathcal{H}_{V}$. However, the well established substitution is rather

$$
z_{\sigma}=e^{\dagger}\left(1-p_{\sigma}^{\dagger} p_{\sigma}\right)^{-\frac{1}{2}}\left(1-e^{\dagger} e-p_{-\sigma}^{\dagger} p_{-\sigma}\right)^{-\frac{1}{2}} p_{\sigma}
$$

which reproduces the $U=0$ spinless case and the Gutzwiller approximation ${ }^{3}$ in the saddle point evaluation and which led to the excellent agreement of the SBMF with numerical simulations in the case of the single band Hubbard model. We note that $\mathcal{H}_{V}$ has the same matrix elements in the physical subspace for any of these representations for $z_{\sigma}$.

In the third step of the scheme the bosonic fields, including the Lagrange multipliers, are replaced by their respective saddle point values. Here we emphasize that $z_{\sigma}^{2}$ is in fact the quasiparticle weight, as may be confirmed by straightforward evaluation of $\frac{\partial \Sigma(\omega)}{\partial \omega}=1-\frac{1}{z_{\sigma}^{2}}$, where $\Sigma(\omega)$ represents the impurity site self-energy.

Before we discuss the KR SBMF results, the renormalization of the nonlocal Coulomb interaction $I$ has to be introduced:

$$
\mathcal{H}_{I}=I n_{\mathrm{d}} n_{\mathrm{c}}=I \sum_{\sigma}\left(1-y_{\sigma}^{\dagger} y_{\sigma}\right) n_{\mathrm{c}, \sigma}
$$

The natural choice would be $y_{\sigma}=e$ (spin independent), as $\left(1-e^{\dagger} e\right)$ is the density operator. However, on the 
saddle point level, we rather expect that $y_{\sigma}=z_{\sigma}^{\dagger}$ should account for a more appropriate representation. As $z_{\sigma}^{\dagger} z_{\sigma}$ is related to the quasiparticle weight, $1-y_{\sigma}^{\dagger} y_{\sigma}$ characterizes the incoherent part of an electronic excitation. It here refers to a local process, Eq. (8). Correspondingly we define:

$$
y_{\sigma}^{\dagger}=e^{\dagger}\left(1+\varepsilon-p_{\sigma}^{\dagger} p_{\sigma}\right)^{-\frac{1}{2}}\left(1-e^{\dagger} e-p_{-\sigma}^{\dagger} p_{-\sigma}\right)^{-\frac{1}{2}} p_{\sigma} .
$$

An infinitesimal convergence factor $\varepsilon>0$ has been introduced to ensure the property $y_{\sigma}=0$ for $e=0$, also on the saddle point level. This singular assignment of $y_{\sigma}=0$ may occur if the hybridization is suppressed either due to the formation of a triplet state or on account of the special choice $V=0$. Otherwise $\varepsilon$ may be set to zero from the outset. Such a convergence factor is not necessary for $z_{\sigma}$ in the hybridization, Eq. (6). We emphasize again that replacing $I n_{\mathrm{d}} n_{\mathrm{c}}$ by the expression on the rhs of Eq. (8) with $y_{\sigma}^{\dagger}$ from Eq. (9) does not hurt the correctness of the representation, i.e., all matrix elements of $\mathcal{H}_{I}$ are unaffected in the physical subspace. Moreover, by extending the scheme developped in Refs. 29, 30] to the KR representation, it can be shown by direct evaluation of the path integral that the exact partition function is recovered when using Eqs. (8, 9).

$K R S B M F$. The saddle point evaluation is now easily implemented: (i) the bosonic fields are replaced by real variables and (ii) these variables are found from the minimization of the grand canonical potential. With (i) we identify the fermionic matrix as:

$$
\left[\mathcal{E}_{\sigma}\right]=\left(\begin{array}{cc}
E_{\mathrm{c}, \sigma} & z_{\sigma} V \\
z_{\sigma} V & E_{\mathrm{f}, \sigma}
\end{array}\right) .
$$

with $E_{\mathrm{c}, \sigma}=\epsilon_{\mathrm{c}}+\left(1-y_{\sigma}^{2}\right) I-\mu$ and $E_{\mathrm{f}, \sigma}=\epsilon_{\mathrm{d}}+\lambda_{\sigma}-\mu$. Its eigenvalues are

$$
E_{ \pm, \sigma}=\frac{1}{2}\left(E_{\mathrm{c}, \sigma}+E_{\mathrm{f}, \sigma} \pm \sqrt{\left(E_{\mathrm{c}, \sigma}-E_{\mathrm{f}, \sigma}\right)^{2}+4 z_{\sigma}^{2} V^{2}}\right)
$$

For convenience, we have introduced a joint chemical potential $\mu$ for all electrons (on the $d$ - and the $c$-site) to control the filling.

Part (ii) of the evaluation depends crucially on the number of electrons $N$ is the system. We first consider the two-electron case $(N=2)$. The free energy $F$ reads: $F(N=2, T)=-T \sum_{\sigma, r= \pm} \ln \left(1+\exp \left(-\beta E_{r, \sigma}\right)\right)-$ $\sum_{\sigma} \lambda_{\sigma} p_{\sigma}^{2}-\alpha\left(1-e^{2}-\sum_{\sigma} p_{\sigma}^{2}\right)+2 \mu$ where the first log-term is standard and the terms with the Lagrange multipliers $\lambda_{\sigma}$ and $\alpha$ were generated through the constraints. The phases of the slave boson fields do not enter into the saddle point evaluation, so $e$ and $p_{\sigma}$ are now real numbers, as well as $\alpha$ and $\lambda_{\sigma}$. The quadratic term $e^{2}$ is to be identified with the hole number $x$ on the correlated site, i.e., $x=e^{2}$. We take the temperature $T$ in units of $k_{B}$ and $\beta=1 / T$. For the limit $T \rightarrow 0$ we have $F(N=2,0)=$ $\sum_{\sigma} E_{-, \sigma}-\sum_{\sigma} \lambda_{\sigma} p_{\sigma}^{2}+\alpha\left(e^{2}+\sum_{\sigma} p_{\sigma}^{2}-1\right)+2 \mu$. The minimization of the ground state $F(N=2,0)$ is cumbersome but straightforward. We obtain:

$$
\begin{aligned}
& F(N=2, T=0)-2 \epsilon_{\mathrm{d}}= \\
& \quad(\Delta(1+x)+I(1-x)-2 V \sqrt{2 x} \sqrt{1-x})
\end{aligned}
$$

Here, the hole number on the correlated site obeys the analytical relation (with $\nu=-1$ and $x_{-}=x$ ):

$$
x_{\nu}=\frac{1}{2}\left(1+\nu \frac{\Delta-I}{\sqrt{(\Delta-I)^{2}+8 V^{2}}}\right),
$$

which displays a monotonous decay with increasing $(\Delta-$ $I) / V$. The ground state is paramagnetic and, with $p_{\sigma}^{2}=p_{-\sigma}^{2} \equiv p^{2}$, the constraint yields the correct relation $p^{2}=(1-x) / 2$ for the expectation value of single occupancy on the correlated site. Notably, these relations, Eqs. (12]13), are exact. This statement is consistent with the observation that the lowest two-particle state acquires the energy $\left(E_{-, \uparrow}+E_{-, \downarrow}\right)=E_{-}^{(s)}$ of the lower antiferromagnetic singlet, Eq. (3), for the saddle point solution of the bosonic fields.

However, it is not only the lowest energy state in the two particle sector, which is solved exactly in the KR SBMF scheme. The approach also provides the exact solutions for the single particle $(N=1)$ and the three particle $(N=3)$ sectors, and moreover the triplet solution for $(N=2)$. These latter cases are not as surprising as the two-particle solution for the paramagnetic ground state. Nevertheless, these states will be important for the calculation of correlation functions.

Here it suffices to emphasize that the KR approach was set up with the proviso that the spin polarized limit is correctly implemented through the weight factors $z_{\sigma}{ }^{3}$ In fact, we find for $N=1$ the hole expectation value on the correlated site $x=\frac{1}{2}\left(1-\Delta / \sqrt{\Delta^{2}+4 V^{2}}\right)$ and the fermionic matrix Eq. (10) reduces to the simple $2 \times 2$ matrix with entries $\epsilon_{\mathrm{c}}$ and $\epsilon_{\mathrm{d}}$ on the diagonal and $V$ as the off-diagonal matrix elements. This represents correctly a two-level problem. The $N=2$ triplet state and the $N=3$ case are exactly reproduced because these are local states with vanishing kinetic term $\mathcal{H}_{V}$ and the weight factors $y_{\sigma}$ for the nearest neighbor interaction $I$ have been chosen suitably in Eq. (9). Truly, for the triplet state, we could verify that $\left(E_{-, \uparrow}+E_{+, \uparrow}\right)=E^{(t)}$ for $E_{ \pm, \uparrow}$ from the corresponding saddle point solution. There, the solution becomes the ground state if the model free energy is extended by a magnetic term, linear in the external field.

The high energy singlet state presents, for the limit $V \rightarrow 0$, a local singlet with the two electrons on the uncorrelated site. For $V \neq 0$, this singlet becomes non-local as the electronic states hybridize with the correlated site. It is important to observe that the KR SBMF scheme is flexible enough to accommodate also this second nonlocal two-particle state correctly. In order to gain the exact expression $E_{+}^{(s)}$ of Eq. (3) , the saddle point evaluation for the bosonic fields has to be retraced, however, with $\left(E_{+, \uparrow}+E_{+, \downarrow}\right)$ for the two-particle state. We find for the hole number on the correlated site $x=x_{+}$(see 
Eq. (13) $)$ and $\left(E_{+, \uparrow}+E_{+, \downarrow}\right)=E_{+}^{(s)}$. In other words, we here minimize the energy in the subspace of the Hilbert space that is orthogonal to the ground state.

Spin Correlation function. The spin correlation function involves transitions between states in the singlet and triplet spin sector which are separated by $E^{(t)}-E_{-}^{(s)}$. For the two-site model we can easily evaluate the spin correlation function $\left\langle\phi_{ \pm}^{(s)}\left|S_{+}(t) S_{-}(0)\right| \phi_{ \pm}^{(s)}\right\rangle$ where $\phi_{ \pm}^{(s)}$ refers to the singlet states and $S_{-}(0)=d_{\downarrow}^{\dagger} d_{\uparrow}=S_{+}^{\dagger}(0)$ acts on the correlated (impurity) site. The singlet state ket-vector is:

$$
\left|\phi_{ \pm}^{(s)}\right\rangle=a\left(c_{\uparrow}^{\dagger} c_{\downarrow}^{\dagger}\right)|0\rangle \pm b\left(d_{\uparrow}^{\dagger} c_{\downarrow}^{\dagger}+c_{\uparrow}^{\dagger} d_{\downarrow}^{\dagger}\right)|0\rangle
$$

where the fermionic operators act on their vacuum state $|0\rangle$, and the normalization factors are $a=\sqrt{x}$ and $b=$ $\sqrt{\frac{1}{2}(1-x)}$. In fact, the total spin operator $S_{+/-}^{\text {(tot })}$ for the two sites correctly yields the singlet state property $S_{+--}^{(\text {tot })}\left|\phi_{ \pm}^{(s)}\right\rangle=0$. The spin correlation function for the correlated site is, e.g., for $T \ll E^{(t)}-E_{-}^{(s)}$ and $\Delta-I>$ 0 , and for a chemical potential which fixes the particle number to two:

$$
\left\langle\phi_{-}^{(s)}\left|e^{-\beta \mathcal{H}} e^{-i t \mathcal{H}} d_{\uparrow} d_{\downarrow}^{\dagger} e^{i t \mathcal{H}} d_{\downarrow} d_{\uparrow}^{\dagger}\right| \phi_{-}^{(s)}\right\rangle
$$

The procedure for its evaluation in the $\mathrm{KR}$ technique is straightforward: the exponential Hamiltonian terms are taken in their respective mean field form which act on the states generated by the spin operators. As the saddle point evaluation delivers the exact energy levels, we identify:

$$
\left\langle S_{+}(t) S_{-}(0)\right\rangle=\frac{1}{2}(1-x) e^{i t\left(E^{(t)}-E_{-}^{(s)}\right)}
$$

where the partition function $Z=e^{-\beta E_{-}^{(s)}}$ cancels the temperature factor. Similarly, the exact spin correlation function with a trace, which includes also the triplet and high energy singlet, is recovered for arbitrary temperature values.

It is the remarkable flexibility of the slave boson technique which correctly renders certain limits of strongly correlated electron systems. This adaptability originates in the freedom to choose appropriate weight factors for the interaction terms in the projected field theory. For the two-site SIAM, the generic choice of this weight factors already leads to the exact solution of the model. The choice is generic in the sense that it was introduced early on for the Hubbard model in the original work on the KR slave boson scheme.$^{\frac{3}{}}$ It is striking to observe that even the inclusion of a non-local Coulomb repulsion in this model does not break the exactitude of the saddle point evaluation in this scheme.

Acknowledgments. Illuminating discussions with P. Wölfle are gratefully acknowledged. The work was supported by DFG through SFB 484. T. K. is grateful for the kind hospitality at the CRISMAT in Caen and at the Aspen Center for Physics where part of this work has been accomplished.
1 See, e.g., the recent reviews P. A. Lee, N. Nagaosa, and X. G. Wen, Rev. Mod. Phys. 7817 (2006); S. Maekawa et. al., Physics of Transition Metal Oxides (Springer Verlag, Berlin, 2004).

2 S. E. Barnes, J. Phys. F: Metal Phys. 6, 1375 (1976); ibid. 7, 2637 (1977).

${ }^{3}$ G. Kotliar and A. E. Ruckenstein, Phys. Rev. Lett. 57, 1362 (1986).

4 R. Frésard and P. Wölfle, Int. J. Mod. Phys. B 6, 685 (1992); Erratum, Int. J. Mod. Phys. B 6, 3087 (1992).

${ }^{5}$ R. Frésard, G. Kotliar, Phys. Rev. B 56, 12909 (1997); H. Hasegawa, ibid. 56, 1196 (1997).

${ }^{6}$ R. Frésard, M. Dzierzawa, and P. Wölfle, Europhys. Lett. 15, 325 (1991).

7 W. Zimmermann, R. Frésard, and P. Wölfle, Phys. Rev. B 56, 10097 (1997).

8 S. Florens, A. Georges, G. Kotliar, and O. Parcollet, Phys. Rev. B 66, 205102 (2002).

9 W. Metzner, and D. Vollhardt, Phys. Rev. Lett. 62, 324 (1989); W. Metzner, Z. Phys. B 77253 (1989); W. Metzner, and D. Vollhardt, Phys. Rev. B 37, 7382 (1988).

10 W.F. Brinkman and T.M. Rice, Phys. Rev. B 24302 (1970).

11 R. Frésard and T. Kopp, Nucl. Phys. B 594, 769 (2001).

12 P. Coleman, Phys. Rev. B 29 (1984) 3035.

13 N. Read and D. M. Newns, J. Phys. C 16, 3273 (1983).

14 Z. Tesanovic and O. T. Valls, Phys. Rev. B 34, 1918 (1986).
15 K. Harigaya, J. Phys: Condens. Matter 2, 3259 (1990).

16 C. Pépin, Phys. Rev. Lett. 98, 206401 (2007).

17 N. Pavlenko and T. Kopp, Phys. Rev. Lett. 97, 187001 (2006).

18 A. Rüegg, S. Pilgram, and M. Sigrist, Phys. Rev. B 75, 195117 (2007)

19 L. Lilly, A. Muramatsu, and W. Hanke, Phys. Rev. Lett. 65, 1379 (1990).

${ }^{20}$ Q. Yuan and T. Kopp, Phys. Rev. B 65, 085102 (2002).

21 G. Seibold, E. Sigmund, and V. Hizhnyakov, Phys. Rev. B 57, 6937 (1998).

22 M. Raczkowski, R. Frésard, and A. M. Oleś, Phys. Rev. B. 73, 174525 (2006).

${ }^{23}$ Y. Bang et. al., Int. J. of Mod. Phys. B 6, 531 (1992).

24 E. Koch, Phys. Rev. B 64, 165113 (2001).

25 V. Zlatic et. al., Phys. Rev. B 68, 104432 (2003).

26 Th. Jolicœur, and J. C. Le Guillou, Phys. Rev. B 44, 2403 (1991).

27 E. Arrigoni et. al., Phys. Rep. 241, 291 (1994).

28 S. Kirchner, J. Kroha, and P. Wölfle, Phys. Rev. B 70, 165102 (2004).

29 R. Frésard, H. Ouerdane, and T. Kopp, Nucl. Phys. B 785, 286 (2007).

30 R. Frésard, H. Ouerdane, and T. Kopp, Europhys. Lett. 82, 31001 (2008). 